\title{
Chronic Kidney Disease and $H$. pylori Prevalence: A Significant Association?
}

\author{
Mahboube Ganji-Arjenaki ${ }^{1} \cdot$ Mahmoud Rafieian-Kopaei $^{1}$
}

Published online: 9 June 2017

(C) Springer Science+Business Media, LLC 2017

The Wijarnpreecha et al. [1] study, entitled "Association of Helicobacter pylori with Chronic Kidney Diseases: A Meta-Analysis," published in Digestive Diseases and Sciences, investigated the relationship between $H$. pylori infection and the incidence of chronic kidney disease (CKD) and non-dialysis-dependent kidney disease. Wijarnpreecha et al. reported that the association in question was not significant. Although their work is interesting, we would like to comment on the method of analysis they adopted to conduct this meta-analysis. They analyzed nine cross-sectional studies using $95 \%$ confidence intervals (CI) and odds ratio (OR) to investigate association between H. pylori infection and CKD. The prevalence of odds ratio (POR) method was used to explain the prevalence of $H$. pylori in patients with and without kidney disease, illustrated by two graphs [1]. In cross-sectional studies, the POR can be computed using the same ad/bc formula (POR is a measure of the association between the two factors in the $2 \times 2$ table). POR is calculated as the OR to measure the incidence rate in two groups [2, 3]. In Wijarnpreecha et al.'s meta-analysis, the $p$ value was $>0.05$ ( $p=0.4$ or $0.9)$ for the OR but $<0.05(p=0.0)$ for prevalence. They reported that there was no association between $H$. pylori infection and the two types of kidney disease, though the prevalence was $53 \%$ with $p<0.05$. Since a significant correlation between the prevalence of $H$. pylori and nephropathy in 8 of cited studies existed (Table 1 in Ref. [1]), it would be better to use POR only; in that case, the relationship would be considered significant, and compatibility would be observed between the input and output data. Then, the prevalence in the two subgroups (CKD and non-dialysis-dependent kidney disease) could be illustrated separately using two graphs.

In summary, re-analysis of the data reported by Wijarnpreecha et al. [1] using alternate and arguably superior statistical methods would reveal a significant correlation between CKD and Hp prevalence.

\section{References}

1. Wijarnpreecha $\mathrm{K}$, Thongprayoon $\mathrm{C}$, Nissaisorakarn $\mathrm{P}$, et al. The association of Helicobacter pylori with chronic kidney diseases: a meta-analysis. Dig Dis Sci. (Epub ahead of print). doi.10.1007/ s10620-017-4516-z.

2. Schoenbach VJ, Rosamond WD. Understanding the Fundamentals of Epidemiology: An Evolving Text. Chapel Hill: North Carolina; 2000.

3. Shy DCM. Epidemiology 160/600. Introduction to Epidemiology for Public Health course lectures, 1994-2001. The University of North Carolina at Chapel Hill, Department of Epidemiology.
Mahmoud Rafieian-Kopaei

rafieian@yahoo.com

1 Medical Plants Research Center, Shahrekord University of Medical Sciences, Shahrekord, Iran 